\title{
A fast approach for human action recognition
}

\begin{abstract}
This paper presents a fast approach to represent and recognize human actions. For representation, a feature vector is constructed from spatiotemporal data of silhouettes based on appearance and motion. For classification, a new Radial Basis Function Network (RBF), called Time Delay Input Radial Basis Function Network is proposed by introducing time delay units to the RBF in a novel approach. The proposed network has a few desirable features such as easier learning process and more flexibility. The representational power and speed of the proposed method for action recognition were evaluated using a publicly available dataset. Based on experimental results, implemented in MATLAB and on standard PCs, the average time for constructing a feature vector for a high-resolution video is almost $20 \mathrm{~ms} /$ frame. Furthermore, the proposed approach demonstrates good performance in terms of execution time and overall performance (a new performance measure that combines accuracy and speed into one metric).
\end{abstract}

Keyword: Action recognition; Action representation; Motion descriptor; Neural network; Radial basis function network 